\title{
Serum Midkine and Osteopontin Levels as Diagnostic Biomarkers of Hepatocellular Carcinoma
}

Hossam Hodeib ${ }^{1}$, Ola ELshora ${ }^{1}$, Amal Selim², Nesreen Mohammed Sabry ${ }^{3}$, Heba Mohamed EL-ashry ${ }^{4}$

${ }^{1}$ M.D. in Clinical Pathology, Faculty of Medicine Tanta University, Tanta, Egypt

${ }^{2}$ M.D. in Internal Medicine, Faculty of Medicine Tanta University, Tanta, Egypt

${ }^{3}$ M.D. in Clinical Oncology, Faculty of Medicine Tanta University, Tanta, Egypt

${ }^{4}$ M.D. in Tropical Medicine, Faculty of Medicine Tanta University, Tanta, Egypt

Type of article: Original

\begin{abstract}
Introduction: Hepatocellular Carcinoma (HCC) is a primary tumor of the liver; it is one of the most common cancers worldwide. Osteopontin (OPN) is a phosphorylated glycoprotein which is implicated in enhancing invasive and metastatic progression of many tumors. Midkine (MDK) is a 13-kDa small heparin-binding growth factor which plays a significant role in carcinogenesis related activities. The aim of this study was to assess the efficacy of serum Midkine and Osteopontin levels as diagnostic biomarkers of Hepatocellular Carcinoma.

Methods: This study was carried out from January 2014 to January 2016 in Internal Medicine, Clinical Oncology and Tropical Medicine Departments of Tanta University Hospital (Egypt). One hundred forty subjects were enrolled in our study, they were divided into four groups: Group I included 35 patients presented with HCV without cirrhosis; Group II included 35 patients presented with HCV with liver cirrhosis; Group III included 35 patients presented with HCC on top of cirrhosis; and Group IV included 35 apparently healthy subjects as a control group. The studied groups were age and sex matched. Routine and specific (OPN and MDK) laboratory investigations were performed in all included subjects.

Results: The main finding of the present work was that the mean serum levels of OPN and MDK were significantly elevated in HCC patients either by comparing HCC patients vs. HCV patients without cirrhosis, HCC patients vs. HCV patients with cirrhosis or HCC patients vs. healthy subjects. Interestingly, by performing a ROC analysis, serum MDK levels had better sensitivity and specificity than OPN and AFP levels in the diagnosis of HCC $(98.4 \%, 97.1 \%$ and $97 \%)$ and $(96.2 \%, 95.3 \%$ and $95 \%)$ for MDK, OPN and AFP respectively.

Conclusion: Serum MDK and OPN levels are comparable to AFP levels and could be used as potential diagnostic biomarkers of $\mathrm{HCC}$ in $\mathrm{HCV}$ patients with liver cirrhosis and in the prediction of liver cirrhosis in $\mathrm{HCV}$ patients without cirrhosis.
\end{abstract}

Keywords: Hepatocellular Carcinoma, Midkine (MDK), Osteopontin (OPN)

\section{Introduction}

Hepatocellular Carcinoma (HCC) is a primary tumor of the liver, it is one of the most common cancers worldwide (1), as with other solid malignant tumors, hepatic tumorgenesis is a multistep process due to uncontrolled cellular growth, together with alteration of the blood supply to enhance tumor growth (2). HCC occurs mainly in patients with chronic liver disease and cirrhosis (1). Hepatitis $\mathrm{C}$ virus (HCV) and Hepatitis B virus (HBV) chronic infections are the main causes of HCCs, there are other causes, such as metabolic disorders, alcohol abuse and obesity (3). HCV induced chronic liver injury, causes fibrosis, cirrhosis, and eventually HCC (4). Liver cancer shows no symptoms in the early stage, as clinical symptoms become evident in the late stage, resulting in unsatisfactory curative results, so early diagnosis and effective treatment improve overall survival and disease prognosis of HCC patients (5). Despite advances in diagnostic techniques and therapy, HCC usually ends in fatal outcome (6). Alphafetoprotein (AFP) is the commonly used serological marker to detect HCC, however, it is not satisfactory due to low sensitivity and specificity (7). Therefore, AFP is not sufficient as a seldom tool for screening and diagnosing of

\section{Corresponding author:}

Dr. Hossam Hodeib, Clinical Pathology Department, Faculty of Medicine Tanta University, Tanta, Egypt.

Tel.: +201006023268, Email: hossamhodeib@gmail.com

Received: August 24, 2016, Accepted: November 25, 2016, Published: January 2017

iThenticate screening: November 26, 2016, English editing: December 18, 2016, Quality control: January 04, 2017

(C) 2017 The Authors. This is an open access article under the terms of the Creative Commons Attribution-NonCommercialNoDerivs License, which permits use and distribution in any medium, provided the original work is properly cited, the use is non-commercial and no modifications or adaptations are made. 
HCC. New sensitive markers are needed for early identification to improve clinical outcomes of HCC patients. Osteopontin (OPN), a phosphorylated glycoprotein which is expressed by a variety of tissues such as fibroblast, some bone marrow cells, smooth muscle, and is secreted into body fluids (8). OPN is involved in numerous physiologic functions such as bone remodeling, inflammation and immune function. It is implicated in enhancing invasive and metastatic progression of many tumors by altering activity of matrix metalloproteinases, and PI3KAKT signaling (9). OPN is an important anti-apoptotic factor in many circumstances. It blocks the activation induced cell death of macrophages and T cells (10). There have been studies reporting the use of plasma OPN as a marker for HCC (11-13), but its diagnostic value continues to be a subject of debate, when compared with AFP. Midkine (MDK), a 13-kDa small heparin-binding growth factor. In humans, it is encoded by the MDK gene on chromosome 11 (14). MDK plays a significant role in carcinogenesis related activities, such as proliferation, migration, antiapoptosis, mitogenesis, transformation, and angiogenesis, in many types of solid tumors, including hepatocellular carcinomas $(15,16)$. Therefore our study aimed at evaluating the diagnostic utility of serum OPN and MDK levels, compared to AFP, for the diagnosis of HCC in HCV related liver cirrhosis.

\section{Material and Methods}

\subsection{Research design and participants}

This study was carried out from January 2014 to January 2016 in Internal Medicine, Clinical Oncology and Tropical Medicine Departments, Tanta University Hospital, Egypt. 140 subjects were enrolled in our study; they were divided into four groups: Group I included 35 patients presented with HCV without cirrhosis; Group II included 35 patients presented with HCV with liver cirrhosis; Group III included 35 patients presented with HCC on top of cirrhosis; and Group IV included 35 healthy adult subjects as a control group. The studied groups were age and sex matched.

\subsection{Selection criteria}

Inclusion criteria were patients with age $>18$ years, regarding HCV patients without cirrhosis and HCV patients with cirrhosis, diagnosis was confirmed by clinical examination, laboratory and radiologic investigations. Regarding HCC patients, diagnosis was confirmed by histological examination of hepatic focal lesions. Exclusion criteria were patients suffering from any malignancy other than HCC, inflammatory, and autoimmune conditions.

\subsection{Ethics of research}

The study was approved by the hospital Ethical Committee, and was performed according to the principles of the declaration of Helsinki; a written informed consent of the patients and control subjects was obtained.

\subsection{Data collection and assays}

\subsubsection{Blood Collection and Laboratory Assay}

Laboratory assays were performed on blood obtained from patients, and were determined at Clinical Chemistry and Hematology units, Clinical Pathology Department, Tanta University Hospital, Egypt. Whole blood was collected by standard venipuncture in VACUETTE® Blood Collection Tubes (Greiner Bio-One, Kremsmuenster, Austria) containing $0.109 \mathrm{~mol} / 1(3.2 \%)$ buffered sodium citrate solution, containing K2EDTA, and containing clot activator/Sep. Citrated blood samples were was centrifuged at 2,500 x g for 15 minutes and citrated plasma samples were immediately separated and used for International Normalized Ratio assay on fully automated blood coagulation analyzer (Sysmex CA 1500, Siemens Healthcare Global, Erlangen, Germany). Whole EDTA blood samples were used for complete blood count assay on fully automated cell counter (Erma INC, PCE 210 N, Tokyo, Japan). Whole blood samples were left to clot for 5 to 10 minutes at room temperature and serum samples were separated by centrifugation at $1000 \mathrm{x}$ g for 10 minutes. One part of the serum samples was stored at $-20^{\circ} \mathrm{C}$ untill the time of specific laboratory assay and the other part was immediately used for routine laboratory investigations. Serum samples were used for serum bilirubin, ALT, AST, and albumin assay on fully automated chemistry analyzer (Konelab Prime 60i, Konelab, Vantaa, Finland). AFP was estimated on automated immunoassay analyzers (Tosoh AIA 1800 ST, Tokyo, Japan). OPN levels were measured by Human Osteopontin Platinum ELISA (BMS2066/BMS2066TEN, eBioscience, Vienna, Austria). MDK levels were measured by Human Midkine ELISA (Cat. No.: RD191042200R, BioVendor, Czech Republic).

\subsubsection{Osteopontin Assay}

Human OPN present in the sample or standard, bound to antibodies adsorbed to the microwells (first incubation). Following incubation, unbound biological components were removed by washing and a biotin-conjugated antihuman OPN antibody was added and bound to human OPN, captured by the first antibody (second incubation). Following this step, unbound biotin-conjugated anti-human OPN antibody was removed by washing. Streptavidin- 
HRP was added and bound to the biotin-conjugated anti-human OPN antibody (third incubation). Following this step, unbound Streptavidin-HRP was removed by washing and substrate solution reactive with HRP was added to the wells (fourth incubation). A colored product was formed in proportion to the amount of human OPN present in the sample or standard. The reaction was terminated by addition of acid, and absorbance was measured at $450 \mathrm{~nm}$. A standard curve was prepared and human OPN concentrations were determined.

2.4.3. Midkine Assay

BioVendor Human Midkine ELISA is a sandwich enzyme immunoassay. Incubation of standards and samples was carried out using microplate wells, pre-coated with polyclonal anti-human MDK antibody. After the standards and samples were incubated for 20 minutes at $37^{\circ} \mathrm{C}$ and washed, biotin labelled polyclonal anti-human MDK antibody was added and incubated with captured MDK for one hour. Streptavidin-HRP conjugate was added, following incubation and washing. The remaining HRP conjugate was allowed to react with the substrate solution (TMB) following 30 minutes' incubation and the final washing step. The reaction was halted by addition of acidic solution, and a proportionate colored product was developed in relation to the amount of human MDK present in the sample or standard. Absorbance was measured at $450 \mathrm{~nm}$. A standard curve was prepared and human MDK concentrations were determined.

\subsection{Statistical analysis}

The collected data were organized, tabulated and statistically analyzed by SPSS version 16 (SPSS Inc. Chicago, IL, USA) using descriptive statistics, Chi-square, One way ANOVA, and Pearson's correlation coefficient (r). The Receiver Operating Characteristic (ROC) curve was done, to detect the area under the curve which reflects the sensitivity, the specificity, positive predictive value (PPV), Negative predictive value (NPV), and the accuracy of AFP (ng/ml), OPN ( $\mathrm{ng} / \mathrm{ml})$ and MDK $(\mathrm{ng} / \mathrm{ml})$ as diagnostic biomarkers in the development of HCC in HCV cirrhotic patients. Significance was adopted at $p<0.05$ for interpretation of results of tests (17).

\section{Results}

In total, 140 subjects; 35 patients presented with HCV without cirrhosis (Group I), 28.6\% were females and 71.4\% were males, 35 patients presented with HCV with liver cirrhosis (Group II), 31.4\% were females and 68.6\% were males, 35 patients presented with HCC on top of cirrhosis (Group III), $25.7 \%$ were females and $74.3 \%$ were males and 35 healthy subjects (Group IV) 31.4\% were females and $68.6 \%$ were males. The mean of age (in years) of Group I, Group II, Group III and Group IV were 46.6 $\pm 5.3,51.5 \pm 5.3,49.1 \pm 4.6$, and $48 \pm 4.4$ respectively. There was no significant difference between all groups regarding age and gender $(\mathrm{p}>0.05)$. Results obtained from the present work revealed that $82.85 \%$ HCC patients had lower limb oedema, $45.72 \%$ had ascites and all HCC patients had jaundice, elevated liver enzymes and INR, and decreased HB levels and platelet count (Table 1,2). The main finding of the present work was that the mean serum levels of OPN and MDK in HCV patients without cirrhosis, HCV patients with cirrhosis, HCC patients and healthy subjects were [(89.6 \pm 26.2 and $0.215 \pm 0.096),(161 \pm 42.4$ and $0.393 \pm 0.196),(247.4 \pm 40$ and $1.149 \pm 0.519)$ and $(19.14 \pm 6$ and $0.189 \pm 0.089)]$ respectively. Moreover, serum levels of OPN and MDK were significantly elevated in HCC patients (Group III) either by comparing HCC patients vs. HCV patients without cirrhosis, HCC patients vs. HCV patients with cirrhosis or HCC patients vs. healthy subjects $(\mathrm{p}<0.001)$ (Table 3). Additionally, no significant correlations were obtained between OPN levels and other laboratory parameters in all the studied groups $(\mathrm{p}>0.05)$ (Table 4). Similarly, no significant correlations were obtained between MDK levels and other laboratory parameters in all the studied groups ( $>0.05$ ) (Table 5). Interestingly, OPN levels were significantly correlated with AFP levels $(r=0.432, p<0.001)$, while MDK levels did not correlate with AFP levels ( $r=0.143, p>0.05)$. In addition, by ROC analysis; the best cut-off value of AFP that discriminated $\mathrm{HCV}$ patients without cirrhosis, $\mathrm{HCV}$ patients with cirrhosis and $\mathrm{HCC}$ patients from healthy subjects were 3.5, 3.85 and $80 \mathrm{ng} / \mathrm{ml}$ respectively (Figure 1). Similarly, the best-cut off value of OPN and MDK that discriminated HCV patients without cirrhosis, HCV patients with cirrhosis and HCC patients from healthy subjects were $[(19,72$ and $170 \mathrm{ng} / \mathrm{ml})$ and $(0.15,0.24$ and $0.65 \mathrm{ng} / \mathrm{ml})]$ respectively. Moreover, MDK had better sensitivity and specificity than OPN and AFP in the diagnosis of HCC (98.4\%, 97.1\% and 97\%) and $(96.2 \%, 95.3 \%$ and $95 \%)$ for MDK, OPN and AFP respectively. Moreover, combined analysis of both MDK and AFP yielded a diagnostic value of $(98 \%)$, and combined analysis of both OPN and AFP yielded a diagnostic value of $(97.5 \%)$ in the diagnosis of HCC (Table 5, 6). 


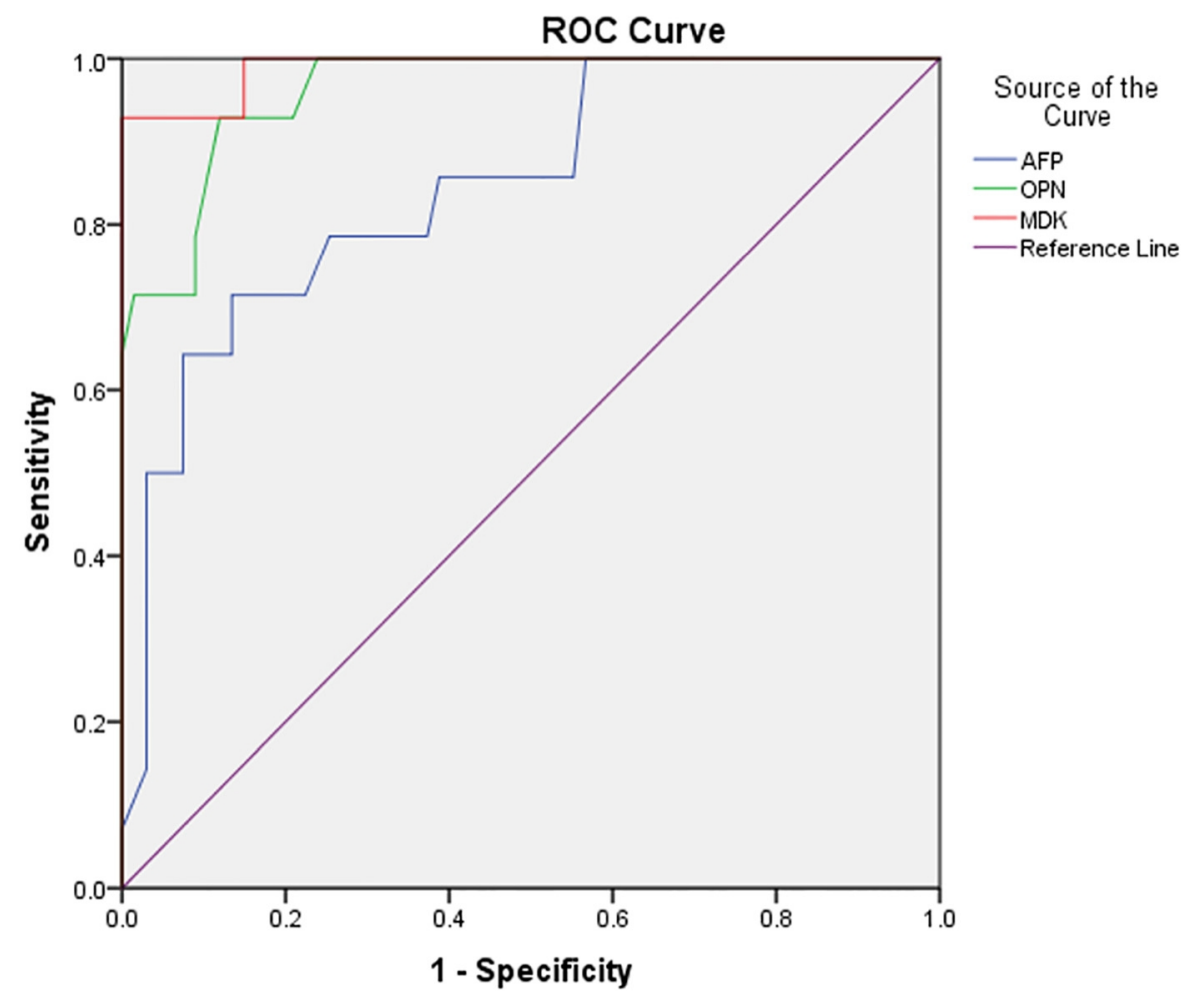

Figure 1. ROC curve of AFP, OPN and MDK in the diagnosis of HCC

Table 1. Clinical data of the studied subjects $(n=140)$

\begin{tabular}{|l|l|l|l|l|l|}
\hline Variable & Group I & Group II & Group III & Group IV \\
\hline \multirow{3}{*}{ Jaundice } & Negative & $29(83.0 \%)$ & $0(0.0 \%)$ & $0(0.0 \%)$ & $35(100.0 \%)$ \\
\cline { 2 - 6 } & Positive & $6(17.0 \%)$ & $35(100.0 \%)$ & $35(100.0 \%)$ & $0(0.0 \%)$ \\
\hline \multirow{3}{*}{ Ascites } & Negative & $35(100.0 \%)$ & $33(94.28 \%)$ & $19(54.28 \%)$ & $35(100.0 \%)$ \\
\cline { 2 - 6 } & Positive & $0(0.0 \%)$ & $2(5.72 \%)$ & $16(45.72 \%)$ & $0(0.0 \%)$ \\
\hline \multirow{2}{*}{ Lower limb oedema } & Negative & $35(100.0 \%)$ & $21(60.0 \%)$ & $6(17.15 \%)$ & $35(100.0 \%)$ \\
\cline { 2 - 6 } & Positive & $0(0.0 \%)$ & $14(40.0 \%)$ & $29(82.85 \%)$ & $0(0.0 \%)$ \\
\hline
\end{tabular}

Table 2. Laboratory data of the studied subjects $(n=140)$

\begin{tabular}{|l|l|l|l|l|}
\hline \multirow{2}{*}{ Variable } & \multicolumn{4}{l|}{ Mean \pm SD } \\
\cline { 2 - 5 } & Group I & Group II & Group III & Group IV \\
\hline Bilirubin $(\mathrm{mg} / \mathrm{dl})$ & $0.9 \pm 0.2$ & $1.9 \pm 0.39$ & $3.7 \pm 1.4$ & $0.7 \pm 0.12$ \\
\hline ALT(U/L) & $42 \pm 15.4$ & $34 \pm 13.2$ & $70.4 \pm 11.8$ & $19.8 \pm 3.7$ \\
\hline AST (U/L) & $47.1 \pm 16.9$ & $39.6 \pm 15$ & $98.5 \pm 16.3$ & $19.14 \pm 3.3$ \\
\hline INR & $1.1 \pm 0.1$ & $1.73 \pm 0.2$ & $2.04 \pm 0.3$ & $1.02 \pm 0.06$ \\
\hline Hb $(\mathrm{gm} / \mathrm{dl})$ & $13 . \pm 81.7$ & $11.5 \pm 1.3$ & $9.8 \pm 0.9$ & $13.8 \pm 1.6$ \\
\hline Albumin $(\mathrm{gm} / \mathrm{dl})$ & $4.35 \pm 0.37$ & $3.06 \pm 0.2$ & $2.54 \pm 0.4$ & $4.21 \pm 0.39$ \\
\hline Platelet $\left(\times 10^{3} / \mathrm{mm}^{3}\right)$ & $205.2 \pm 23.16$ & $129.2 \pm 10.8$ & $108.2 \pm 19.5$ & $234.3 \pm 37.3$ \\
\hline
\end{tabular}

AFP: $\alpha$-fetoprotein; OPN: Osteopontin; Midkine: MDK

Table 3. Plasma levels of AFP, OPN and MDK in the studied subjects $(n=140)$

\begin{tabular}{|c|l|l|l|l|c|}
\hline Variable & Group I & Group II & Group III & Group IV & p-value \\
\hline AFP $(\mathrm{ng} / \mathrm{ml})$ & $6.13 \pm 2.3$ & $7.41 \pm 2.6$ & $683 \pm 188.3$ & $3.64 \pm 1.8$ & $<0.001$ \\
\hline OPN $(\mathrm{ng} / \mathrm{ml})$ & $89.6 \pm 26.2$ & $161 \pm 42.4$ & $247.4 \pm 40$ & $19.14 \pm 6$ & $<0.001$ \\
\hline MDK $(\mathrm{ng} / \mathrm{ml})$ & $0.215 \pm 0.096$ & $0.393 \pm 0.196$ & $1.149 \pm 0.519$ & $0.189 \pm 0.089$ & $<0.001$ \\
\hline
\end{tabular}

AFP: $\alpha$-fetoprotein; OPN: Osteopontin; Midkine: MDK 
http://www.ephysician.ir

Table 4. OPN and other laboratory data in the studied subjects $(\mathrm{n}=140)$

\begin{tabular}{|l|l|l|l|l|l|l|l|l|}
\hline \multirow{2}{*}{ OPN } & \multicolumn{2}{l|}{ Group I } & \multicolumn{2}{l|}{ Group II } & \multicolumn{2}{l|}{ Group III } & \multicolumn{2}{l|}{ Group IV } \\
\cline { 2 - 10 } & $\mathrm{r}$ & $\mathrm{p}$-value & $\mathrm{r}$ & $\mathrm{p}$-value & $\mathrm{r}$ & $\mathrm{p}$-value & $\mathrm{r}$ & $\mathrm{p}$-value \\
\hline Bilirubin & 0.07 & 0.6 & 0.05 & 0.7 & 0.2 & 0.09 & 0.2 & 0.24 \\
\hline ALT & 0.23 & 0.17 & 0.3 & 0.07 & 0.05 & 0.77 & 0.19 & 0.25 \\
\hline AST & 0.24 & 0.16 & 0.3 & 0.05 & 0.08 & 0.63 & 0.25 & 0.14 \\
\hline Albumin & -0.18 & 0.27 & -0.19 & 0.2 & -0.03 & 0.82 & 0.16 & 0.3 \\
\hline INR & 0.08 & 0.6 & 0.9 & 0.5 & 0.32 & 0.05 & 0.03 & 0.8 \\
\hline HB & 0.25 & 0.13 & -0.18 & 0.2 & -0.2 & 0.27 & 0.01 & 0.9 \\
\hline Platelet & -0.05 & 0.7 & -0.16 & 0.3 & -0.23 & 0.17 & 0.19 & 0.25 \\
\hline
\end{tabular}

Table 5. MDK and other laboratory data in the studied subjects $(\mathrm{n}=140)$

\begin{tabular}{|l|l|l|l|l|l|l|l|l|}
\hline \multirow{2}{*}{ MDK } & \multicolumn{2}{|l|}{ Group I } & \multicolumn{2}{l|}{ Group II } & \multicolumn{2}{l|}{ Group III } & \multicolumn{2}{l|}{ Group IV } \\
\cline { 2 - 11 } & $\mathrm{r}$ & $\mathrm{p}$-value & $\mathrm{r}$ & $\mathrm{p}$ - value & $\mathrm{r}$ & $\mathrm{p}$-value & $\mathrm{R}$ & $\mathrm{p}$-value \\
\hline Bilirubin & 0.17 & 0.45 & 0.09 & 0.65 & 0.29 & 0.32 & 0.24 & 0.28 \\
\hline ALT & 0.16 & 0.29 & 0.32 & 0.09 & 0.14 & 0.53 & 0.20 & 0.32 \\
\hline AST & 0.22 & 0.30 & 0.37 & 0.07 & 0.21 & 0.36 & 0.24 & 0.30 \\
\hline Albumin & -0.26 & 0.39 & -0.21 & 0.34 & -0.15 & 0.47 & 0.15 & 0.47 \\
\hline INR & 0.09 & 0.62 & 0.24 & 0.22 & 0.28 & 0.35 & 0.06 & 0.83 \\
\hline HB & 0.11 & 0.53 & -0.18 & 0.25 & -0.20 & 0.26 & 0.16 & 0.42 \\
\hline Platelet & -0.13 & 0.41 & -0.27 & 0.33 & -0.24 & 0.21 & 0.21 & 0.32 \\
\hline
\end{tabular}

Table 6. ROC analysis of AFP, OPN and MDK in the studied subjects

\begin{tabular}{|l|l|l|l|l|l|l|l|l|l|l|}
\hline \multirow{2}{*}{ Variable } & \multicolumn{3}{l}{ HCV without cirrhosis } & \multicolumn{2}{l|}{ HCV with cirrhosis } & \multicolumn{2}{l|}{ HCC } \\
\cline { 2 - 12 } & OPN & AFP & MDK & OPN & AFP & MDK & OPN & AFP & MDK \\
\hline Best cut off value (ng/ml) & 19 & 3.5 & 0.15 & 72.0 & 3.85 & 0.24 & 170 & 80 & 0.65 \\
\hline Sensitivity & $97 \%$ & $94.3 \%$ & $97.5 \%$ & $92 \%$ & $91.4 \%$ & $93.5 \%$ & $97.1 \%$ & $97.0 \%$ & $98.4 \%$ \\
\hline Specificity under the & $60 \%$ & $52.4 \%$ & $62.3 \%$ & $80.3 \%$ & $60.0 \%$ & $85.6 \%$ & $95.3 \%$ & $95.0 \%$ & $96.2 \%$ \\
\hline $\begin{array}{l}\text { Area } \\
\text { curve(AUC) }\end{array}$ & 0.91 & 0.84 & 0.93 & 0.89 & 0.84 & 0.95 & 0.98 & 0.97 & 0.99 \\
\hline
\end{tabular}

\section{Discussion}

To date, about $70 \%$ to $90 \%$ of HCC patients have an established background of chronic liver disease or cirrhosis, which was caused by HBV or HCV infection (18). A large epidemiological study performed on HCC patients in Egypt, revealed that $\mathrm{HCV}$ is the predominant cause of the underlying liver cirrhosis constituting of about $91.32 \%$ of HCC cases, while chronic HVB infection was reported in $2.51 \%$ (19). The only biochemical marker, AFP, is not sufficient enough to identify a substantial proportion of HCC cases. Systematic reviews provided evidence supporting that the use of AFP as a diagnostic and screening test for HCC is limited (20). Results obtained from the present work revealed that $82.85 \%$ HCC patients had lower limb oedema, $45.72 \%$ had ascites and all HCC patients had jaundice, elevated liver enzymes and INR, and decreased HB levels and platelet count. These findings were explained by the progression of the underlying liver cirrhosis with a subsequent decreased albumin and protein synthesis by the liver, and decreased platelet count (due to portal hypertension and splenomegaly). Results of the present work showed that serum levels of OPN and MDK were significantly elevated in HCC patients by comparing these values with that of the other studied groups. These findings were in agreement with Shaheen et al. (21) who found that the median values of the MDK levels in the HCC patients were significantly higher than that of cirrhotic patients and the healthy control subjects. Though the median values of MDK levels in the cirrhotic patients were higher than that in the control group, values did not reach significance. In addition, Fouad et al. (22) reported that there was statistically significant increase in the serum OPN levels in the HCC group, compared to the benign chronic liver disease groups (HCV without cirrhosis, HCV with cirrhosis and Fatty liver disease) and healthy subjects. Moreover, there was no significant difference in the OPN levels between the patients with HCV without cirrhosis and those with fatty liver, and there was statistically significant difference in the serum OPN levels between the healthy control subjects and the other group of patients. Our results showed that OPN levels significantly correlated with AFP levels, while MDK levels did not correlate with AFP levels. In accordance with our results, Fouad et al. (22) reported that there was a positive significant correlation between OPN and AFP, while other studies done by Kim et al. (23) and Nabih et al. (24) have found that the correlation between plasma OPN and serum AFP levels was insignificant. Close to our results, Shaheen et al. (21) confirmed that no significant 
correlation was found between serum MDK levels with tumor size, number or serum levels of AFP. Similarly, Zhu et al. (25) found no significant correlation between MDK levels and aggressive clinicopathologic features such as poor tumor differentiation, microvascular invasion, larger tumor size and advanced tumor stage. ROC analysis illustrated the sensitivity and the specificity of OPN, MDK and AFP. MDK had better sensitivity and specificity than OPN and AFP in the diagnosis of HCC and in the prediction of chronic liver cirrhosis. Moreover, combined analysis of both MDK and AFP yielded a diagnostic value of (98\%) and combined analysis of both OPN and AFP yielded a diagnostic value of (97.5\%). In accordance with our results, Fouad et al. (22) reported that OPN was superior to AFP in the selective detection and diagnosis of HCC and in predicting liver cirrhosis and fatty change. Close to our results, Shaheen et al. (21) found that MDK had similar specificity and better sensitivity than AFP in the diagnosis of HCC. Moreover, MDK had significantly higher specificity than that of AFP only at the cut-off value of $20 \mathrm{ng} / \mathrm{mL}$. The same results were obtained by Zhu et al. (25), who confirmed that MDK had a better performance compared with AFP, in distinguishing early stage hepatocellular carcinomas as well as small hepatocellular carcinomas. Similarly, Vongsuvanh et al. (26) suggested that AFP and MDK may have a complementary role in HCC surveillance and screening. MDK increases the diagnostic yield in AFP-negative HCC, and the presence of either elevated AFP or MDK increases the sensitivity of HCC detection. Our study has several limitations; firstly, the value of MDK in detection of HCV related early HCC, was not analyzed in the present study due to the relative small number of HCC patients, with subsequent inability to classify the patients according to the histological and the clinical staging of HCC, according to the Barcelona Clinic Liver Cancer (BCLC) system. The authors reported different cut-off values of MDK and OPN levels. The exact reason for these differences is not clear, but these discrepancies may be in consequence of the different assay systems and conditions of sample collection used in different studies. Ultimately, most of our HCC patients were at late stage and they were not treated by surgery or other curative therapies without detailed clinical data; therefore, the association between MDK expression and clinicopathologic parameters was not analyzed in this study, which needs to be further investigated later.

\section{Conclusions}

In this study, serum MDK and OPN levels were comparable to those of AFP in HCC group of patients, so serum OPN and MDK may serve as good diagnostic biomarkers for the diagnosis of HCC especially in high risk patients, either OPN or MDK alone or in combination with AFP significantly improve the diagnostic accuracy of HCC with a sensitivity, specificity and accuracy higher than that of AFP alone. Furthermore OPN and MDK were superior to AFP in predicting liver cirrhosis which is risk factor for HCC. Further studies are needed to confirm the diagnostic utility of serum MDK and OPN levels together with AFP in prediction liver cirrhotic patients. Validation of OPN and MDK values either alone or in combination with AFP in HCC diagnosis over larger population of HCC patients is required. Also evaluation of the response of OPN levels to intervention and its relation to prognosis and recurrence of $\mathrm{HCC}$ is recommended.

\section{Acknowledgments:}

The authors would like to thank the nurses at Internal Medicine, Clinical Pathology, Clinical Oncology and Tropical Departments, Tanta University Hospital; for their assistance in conducting the study.

\section{Conflict of Interest:}

There is no conflict of interest to be declared.

\section{Authors' contributions:}

All authors contributed to this project and article equally. All authors read and approved the final manuscript.

\section{References:}

1) Parkin DM, Bray F, Ferlay J, Pisani P. Global cancer statistics, 2002. CA Cancer J Clin. 2005; 55(2): 74108. doi: 10.3322/canjclin.55.2.74. PMID: 15761078.

2) Thorgeirsson SS, Grisham JW. Molecular pathogenesis of human hepatocellular carcinoma. Nat Genet. 2002; 31(4): 339-46. doi: 10.1038/ng0802-339. PMID: 12149612.

3) Sanyal AJ, Yoon SK, Lencioni R. The etiology of hepatocellular carcinoma and consequences for treatment. Oncologist. 2010; 15 Suppl 4: 14-22. doi: 10.1634/theoncologist.2010-S4-14. PMID: 21115577.

4) Farazi PA, DePinho RA. Hepatocellular carcinoma pathogenesis: from genes to environment. Nat Rev Cancer. 2006; 6(9): 674-87. doi: 10.1038/nrc1934. PMID: 16929323.

5) Trinchet JC, Alperovitch A, Bedossa P, Degos F, Hainaut P, Beers BV. Epidemiology, prevention, screening and diagnosis of hepatocellular carcinoma. Bull Cancer. 2009; 96(1): 35-43. doi: 10.1684/bdc.2009.0791. PMID: 19211358. 
6) Farinati F, Marino D, De Giorgio M, Baldan A, Cantarini M, Cursaro C, et al. Diagnostic and prognostic role of alpha-fetoprotein in hepatocellular carcinoma: both or neither? Am J Gastroenterol. 2006; 101(3): 524-32. doi: 10.1111/j.1572-0241.2006.00443.x. PMID: 16542289.

7) Mao Y, Yang H, Xu H, Lu X, Sang X, Du S, et al. Golgi protein 73 (GOLPH2) is a valuable serum marker for hepatocellular carcinoma. Gut. 2010; 59(12): 1687-93. doi: 10.1136/gut.2010.214916. PMID: 20876776.

8) Ashizawa N, Graf K, Do YS, Nunohiro T, Giachelli CM, Meehan WP, et al. Osteopontin is produced by rat cardiac fibroblasts and mediates A(II)-induced DNA synthesis and collagen gel contraction. J Clin Invest. 1996; 98(10): 2218-27. doi: 10.1172/JCI119031. PMID: 8941637, PMCID: PMC507670.

9) Anborgh PH, Mutrie JC, Tuck AB, Chambers AF. Pre- and post-translational regulation of osteopontin in cancer. J Cell Commun Signal. 2011; 5(2): 111-22. doi: 10.1007/s12079-011-0130-6. PMID: 21516514,

10) Standal T, Borset M, Sundan A. Role of osteopontin in adhesion, migration, cell survival and bone remodeling. Exp Oncol. 2004; 26(3): 179-84. PMID: 15494684.

11) Nabih MI, Aref WM, Fathy MM. Significance of plasmaosteopontin in diagnosis of hepatitis C virusrelated hepatocellular carcinoma. Arab J Gastroenterol. 2014; 15(3-4): 103-7. doi: 10.1016/j.ajg.2014.08.002. PMID: 25249230.

12) Wan $\mathrm{HG}, \mathrm{Xu} \mathrm{H}$, Gu YM, Wang H, XuW, Zu MH. Comparison osteopontin vs AFP for the diagnosis of HCC: a meta-analysis. Clin Res Hepatol Gastroenterol. 2014; 38(6): 706-14. doi: 10.1016/j.clinre.2014.06.008. PMID: 25034355.

13) Lee HJ, Yeon JE, Suh SJ, Lee SJ, Yoon EL, Kang K, et al. Clinical utility of plasmaglypican-3 and osteopontin as biomarkers ofhepatocellular carcinoma. Gut Liver. 2014; 8(2): 177-85. doi: 10.5009/gnl.2014.8.2.177. PMID: 24672660, PMCID: PMC3964269.

14) Ibusuki M, Fujimori H, Yamamoto $Y$, Ota K, Ueda M, Shinriki S, et al. Midkine in plasma as a novel breast cancer marker. Cancer Sci. 2009; 100(9): 1735-9. doi: 10.1111/j.1349-7006.2009.01233.x. PMID: 19538527.

15) Kato M, Shinozawa $T$, Kato Sh, Awaya A, Terada T. Increased midkine expression in hepatocellular carcinoma. Archives of Pathology and Laboratory Medicine. 2002; 124(6): 848-85. PMID: 10835519.

16) Muramatsu T. Midkine and pleiotrophin: two related proteins involved in development, survival, inflammation and tumorigenesis. J Biochem. 2002; 132(3): 359-71. doi: 10.1093/oxfordjournals.jbchem.a003231. PMID: 12204104.

17) Dawson BD, Trapp RG. Reading the medical literature: Basic \& Clinical Biostatistics. Lange Medical Book/ McGraw-Hill. Medical Publication Division, New York. 3rd ed. Ch. 7-9, PP 161-218, Ch. 8, PP 21723, Ch. 1, PP 275-281 and Ch. 13, PP 305-314.

18) El-Serag HB, Rudolph KL. Hepatocellular carcinoma: epidemiology and molecular carcinogenesis. Gastroenterology. 2007; 132(7): 2557-76. doi: 10.1053/j.gastro.2007.04.061. PMID: 17570226.

19) Shaker MK, Abdella HM, Khalifa MO, El Dorry AK. Epidemiological characteristics of hepatocellular carcinoma in Egypt: a retrospective analysis of 1313 cases. Liver Int. 2013; 33(10): 1601-6. doi: 10.1111/liv.12209. PMID: 23714212.

20) Colli A, Fraquelli M, Conte D. Alpha-fetoprotein and hepatocellular carcinoma. Am J Gastroenterol. 2006; 101(8): 1939; author reply 1940-1. doi: 10.1111/j.1572-0241.2006.00684_3.x. PMID: 16928257.

21) Shaheen KY, Abdel-Mageed AI, Safwat E, AlBreedy AM. The value of serum midkine level in diagnosis of hepatocellular carcinoma. Int J Hepatol. 2015; 2015: 146389. doi: 10.1155/2015/146389. PMID: 25737783, PMCID: PMC4337261.

22) Fouad SA, Mohamed NA, Fawzy MW, Moustafa DA. Plasma Osteopontin Level in Chronic Liver Disease and Hepatocellular Carcinoma. Hepat Mon. 2015; 15(9): e30753. doi: 10.5812/hepatmon.30753. PMID: 26500684 , PMCID: PMC4612688.

23) Kim J, Ki SS, Lee SD, Han CJ, Kim YC, Park SH, et al. Elevated plasma osteopontin levels in patients with hepatocellular carcinoma. Am J Gastroenterol. 2006; 101(9): 2051-9. doi: 10.1111/j.15720241.2006.00679.x. PMID: 16848813.

24) Nabih MI, Aref WM, Fathy MM. Significance of plasma osteopontin in diagnosis of hepatitis C virusrelated hepatocellular carcinoma. Arab J Gastroenterol. 2014; 15(3-4): 103-7. doi: 10.1016/j.ajg.2014.08.002. PMID: 25249230.

25) Zhu WW, Guo JJ, Guo L, Jia HL, Zhu M, Zhang JB, et al. Evaluation of midkine as a diagnostic serum biomarker in hepatocellular carcinoma. Clin Cancer Res. 2013; 19(14): 3944-54. doi: 10.1158/10780432.CCR-12-3363. PMID: 23719264.

26) Vongsuvanh R, van der Poorten D, Iseli T, Strasser SI, McCaughan GW, George J. Midkine Increases Diagnostic Yield in AFP Negative and NASH-Related Hepatocellular Carcinoma. PLoS One. 2016; 11(5): e0155800. doi: 10.1371/journal.pone.0155800. PMID: 27219517, PMCID: PMC4878793. 